\title{
Src Kinase Inhibitor KX2-391 Ointment
}

National Cancer Institute

\section{Source}

National Cancer Institute. Src Kinase Inhibitor KX2-391 Ointment. NCI Thesaurus. Code C120210.

An ointment containing an inhibitor for both Src tyrosine kinase and tubulin polymerization, with potential antineoplastic activity. Unlike other Src kinase inhibitors which bind to the ATP-binding site, Src kinase inhibitor KX2-391 binds to the peptide substrate binding site of Src kinase, upon topical application. This inhibits both downstream signaling and the proliferation of tumor cells overexpressing Src. Src tyrosine kinase, a non-receptor tyrosine kinase upregulated in many tumor cell types, plays an important role in tumor cell proliferation, motility, invasiveness and survival. KX2391 also binds to tubulin heterodimers and inhibits microtubule polymerization, which disrupts microtubule formation and mitosis, leading to further inhibition of cell proliferation. In addition, KX2-391 inhibits T-cell migration. 